\title{
Updating the Surgical Management of Peritoneal Carcinomatosis in Patients with Neuroendocrine Tumors
}

\author{
Louis de Mestier $^{a}$ Sophie Lardière-Deguelte ${ }^{b}$ Hedia Brixi $^{a} \quad$ Dermot O'Toole $^{d}$ \\ ${\text { Philippe Ruszniewskic }{ }^{c} \text { Guillaume } \text { Cadiot }^{a} \text { Reza Kianmanesh }}^{b}$ \\ Departments of a Hepato-Gastroenterology and Digestive Oncology and ${ }^{\mathrm{b}}$ General, Digestive and Endocrine Surgery, \\ Robert-Debré University Hospital, Reims, and 'Department of Gastroenterology and Pancreatology, Beaujon \\ University Hospital, Clichy, France; ${ }^{d}$ Department of Gastroenterology, St James's Hospital and Neuroendocrine \\ Tumour Clinic, St Vincent's University Hospital and Trinity College, Dublin, Ireland
}

\section{Key Words}

Peritoneal carcinomatosis · Neuroendocrine

tumors - Metastases - Prognosis - Cytoreductive surgery .

Hyperthermic intraperitoneal chemotherapy

\begin{abstract}
Well-differentiated digestive neuroendocrine tumors (NET) are a heterogeneous group of neoplasms usually associated with slow growth but a high rate of metastases, including peritoneal carcinomatosis (PC). Herein, we aimed to comprehensively review the current knowledge of PC in terms of implications for the management and prognosis of patients with NET, including the latest studies and expert statements. NET-derived PC concerns about $17 \%$ of NET patients and up to $30 \%$ of those with small intestine primary NET. It has an independent pejorative prognostic impact. The extent of PC in NET patients and its severity can be expressed by analogy to other malignancies. However, it must be placed in the context of NET disorders, which usually vary from other PCrelated malignancies. Recently, a gravity PC score was proposed by a consensus European Neuroendocrine Tumor Society (ENETS) expert group, but it requires validation. In ad-
\end{abstract}

dition, the form of peritoneal involvement (nodular or fusiform/infiltrative) might influence its prognosis and management. Aggressive surgical management seems justified for subsets of NET-related PC but requires careful selection of the candidates most likely to benefit. Cytoreductive surgery prolongs survival, especially when the peritoneal lesions are completely resected. Too little is known about the benefit of hyperthermic intraperitoneal chemotherapy for NET-derived PC, but if it confers an advantage, it would have to be counterbalanced by its high morbidity.

(c) 2015 S. Karger AG, Basel

\section{Introduction}

Digestive neuroendocrine tumors (NET) are a heterogeneous group of neoplasms sharing clinical, histological and evolutionary features. Well-differentiated NET are usually associated with slow growth and a high rate of metastases. The most common dissemination sites include the liver (in 50-60\% of patients), distant lymph nodes (20-30\%), lungs (3-5\%) and bones $(1-6 \%)[1,2]$. Peritoneal carcinomatosis (PC) develops in $10-33 \%$ of

\section{KARGER 125}

C) 2015 S. Karger AG, Base

0028-3835/15/1012-0105\$39.50/0

E-Mail karger@karger.com

www.karger.com/nen
Reza Kianmanesh

Department of General, Digestive and Endocrine Surgery Robert-Debré University Hospital

125, avenue du Général Koenig, FR-51100 Reims Cedex (France)

E-Mail rkianmanesh@chu-reims.fr 
Table 1. Gilly's score of PC [11]

\begin{tabular}{ll}
\hline Stage & Criteria \\
\hline 0 & $\begin{array}{l}\text { No macroscopic lesions } \\
\text { Granulations }<5 \mathrm{~mm} \text { in size, localized in one part of the }\end{array}$ \\
2 & $\begin{array}{l}\text { abdomen } \\
3\end{array}$ \\
4 & Nodules $5-20 \mathrm{~mm}$ in size, localized or diffuse \\
Macronodules $>20 \mathrm{~mm}$ in size, localized or diffuse
\end{tabular}

patients with digestive NET [2-8]. The authors of the two largest and most recent studies reported similar frequencies of approximately $17 \%[1,9]$. PC is more prevalent in patients with ileal/appendicular than in those with pancreatic NET; it was even suggested that PC in NET could be indicative of an ileal/appendicular origin $[4,7]$.

In 2010, the European Neuroendocrine Tumor Society (ENETS) published consensus guidelines for the management of NET-associated PC [10]. However, recommendations for its surgical management were mainly built on what has been shown for other malignancies, particularly considering cytoreductive surgery (CRS) and hyperthermic intraperitoneal chemotherapy (HIPEC). Several specific studies and expert statements have been published since then. Although NET are heterogeneous malignancies, recent insights may help identify which NET patients might be good candidates for PC surgery. This review was undertaken to comprehensively evaluate the current state of knowledge pertaining to PC in NET and, in particular, its management and prognosis.

\section{Evaluation of PC}

Several scores have been developed to evaluate the extent of PC [11]. The Peritoneal Carcinomatosis Index (PCI), based on the intraoperative quantification of the size of peritoneal lesions $(<0.5 \mathrm{~cm}=1$ point; $0.5-5 \mathrm{~cm}=$ 2 points; $>5 \mathrm{~cm}=3$ points) and the involvement in each of the 12 abdominal-pelvic regions, ranges from 1 to 39 [12]. The PCI is a relevant tool able to predict the completeness of PC surgical cytoreduction. For PC arising from colorectal adenocarcinoma, a PCI $\geq 16$ predicts incomplete surgical removal [13]. For NET, the corresponding threshold was estimated to be 20 [14]. The classification of Gilly et al. [11] also relies on the size of PC nodules and their localization as assessed intraoperatively, and defines 5 stages from 0 to 4 (table 1). Stages 3 and
4 define advanced macroscopic disease and are associated with poorer prognoses. Interestingly, it may be possible to estimate this score on radiological images.

To date, those classifications have not been properly validated for NET patients, and their description of PC resectability leaves much to be desired. Indeed, the evaluation of the PC extent must also take into account the presence of associated lymph nodes and mesenteric retraction, which is frequent in NET-related PC. Peritoneal infiltration can harbor one or several forms comprising bulky, nodular (macro- or micronodular) and/or around nodular fusiform/infiltrative along vascular axes (fig. 1) [15]. Moreover, it can include distant dissemination and/ or lesions contiguous to the primary tumor or large lymph nodes [10].

Notably, those scores are easy to use and reproducible, and seem to evaluate the PC extent appropriately, especially before and after any surgical $\mathrm{PC}$ resection to predict its effectiveness $[10,14,15]$. However, they may no longer be the most relevant, because they require careful intraoperative assessment, and most patients with PC do not undergo surgery. Although the currently available radiological means (i.e., computed tomography scan, somatostatin receptor scintigraphy and magnetic resonance imaging) are widely used to preoperatively evaluate NET patients with PC, their abilities to evaluate the presence of PC or its extent seem poor, and their relationship to intraoperative evaluation has not yet been explored [16]. Finally, laparoscopic staging might be helpful for the initial PC workup, especially to determine its form, but has never been assessed for digestive NET [14]. Laparoscopy enables the detection of PC and the evaluation of its extent, even when microscopic involvement is not detectable on conventional imaging, and could prevent nontherapeutic open surgical interventions $[17,18]$.

\section{Prognostic Impact of Digestive NET-Derived PC}

The prognostic value of PC in digestive NET has been controversial. Its frequent association with liver metastases, which are a major prognostic factor, hampers the assessment of its specific prognostic impact on NET. On the one hand, PC can be asymptomatic, discovered fortuitously, and may not affect prognosis [3, 19]. Conversely, PC may be responsible for abdominal pain and/or obstruction as well as the cause of $40 \%$ of patients' deaths if left untreated [4]. Based on a large retrospective study on 603 patients with ileal NET, in whom PC was a significant independent poor-prognosis factor (hazard ratio, 1.76; 
Table 2. Main studies that evaluated the prognostic impact of PC and its surgical treatment in NET patients

\begin{tabular}{lllrl}
\hline Study & Period & Treatment & Patients, n & 5-year overall survival \\
\hline Norlén et al. [9] & $1985-2010$ & $\begin{array}{l}\text { Conventional surgery: } \\
\text { without PC }\end{array}$ & 501 & $79 \%$ \\
& & with PC & 102 & $52 \%$ \\
\hline Boudreaux et al. [19] & $1998-2004$ & With PC, CRS more or less complete & 82 & $49 \%$ \\
\hline Elias et al. [4] & $1993-2003$ & With PC: & 20 & $41 \%$ \\
& & incomplete CRS & 17 & $66 \%$ \\
\hline Elias et al. [31] & complete CRS+HIPEC & With PC, complete CRS with/without HIPEC & 41 & $69 \%$ \\
\hline
\end{tabular}

Fig. 1. Schematic representation of the different forms of PC dissemination of small bowel NET. The primary tumor (T) is generally unique in the ileum, with regional lymph nodes $(\mathrm{N})$. a No PC. b Diffuse nodular PC (distant and/or contiguous spreading). c Fusiform infiltration of vascular axes (possible contiguous spreading). d Mixed form.

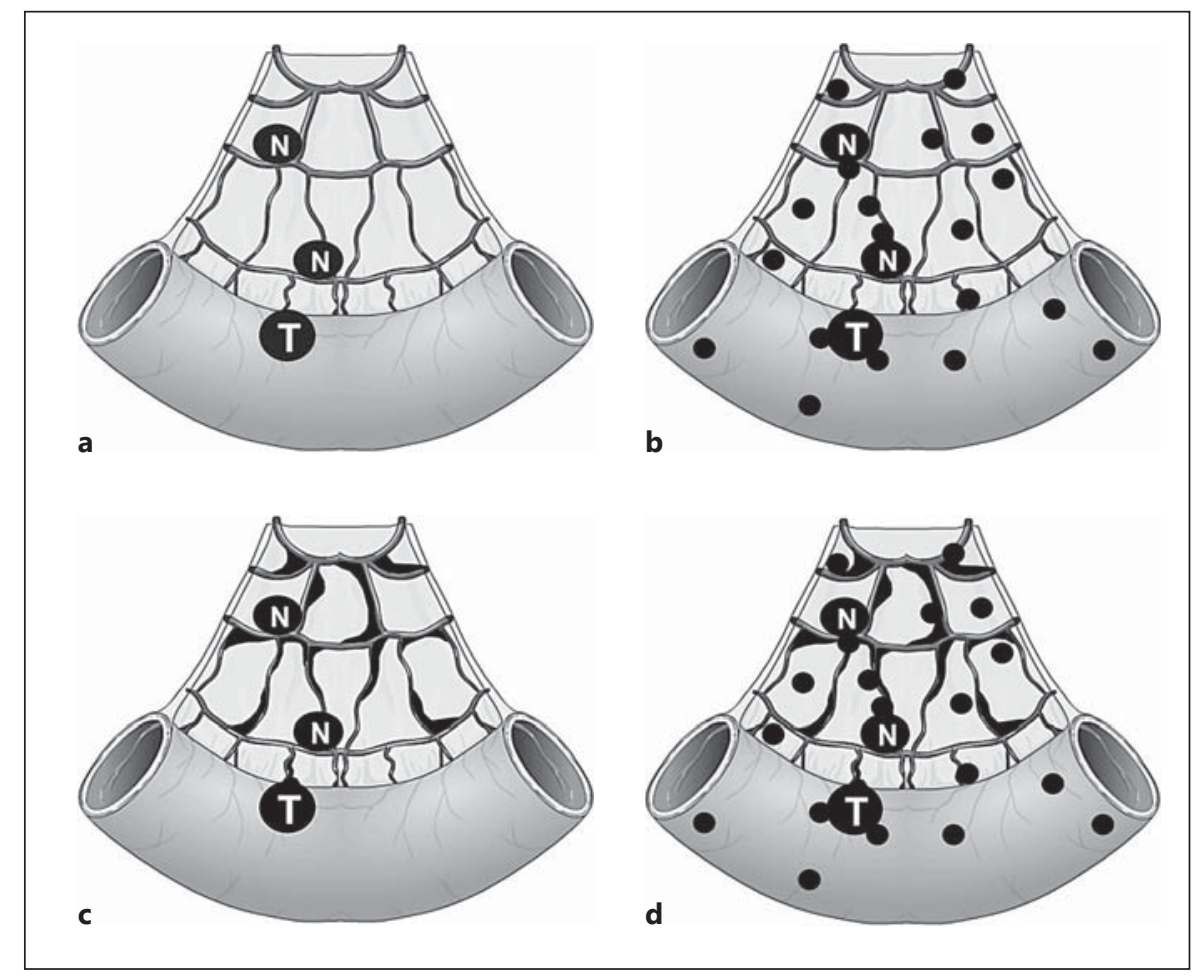

95\% confidence interval, 1.26-2.45), the 5- and 10-year overall survival rates were 52 and $32 \%$, and 79 and $54 \%$ for patients without or with PC, respectively (table 2) [9]. Hence, surgical resection of primary ileal/appendicular NET should systematically be combined with excision of early local-regional peritoneal lesions (including extended lymphadenectomy), with the aim of preventing later complications associated with PC dissemination $[9,20-$ 23].

Taking into account the specificities of PC originating from digestive NET, the ENETS proposed the gravity PC score (GPS), which is a new tool for evaluating the prognosis of patients with NET-derived PC that takes into consideration the presence of lymph node and liver metastases [10] (table 3). Pertinently, assuming that Gilly's score might be estimated on radiological imaging, the GPS could perhaps be calculated for all patients, including those who do not undergo surgery. However, the GPS has not yet been evaluated prospectively. Moreover, the prognostic impact of the type of peritoneal dissemination, i.e., nodular, infiltrative or mixed, warrants exploration in future studies. Beyond a hypothetical prognostic 
Table 3. ENETS GPS for NET with peritoneal metastases (from Kianmanesh et al. [10])

\begin{tabular}{lllll}
\hline & 0 points & 1 point & 2 points & 3 points \\
\hline Lymph node metastases & Localized to the first relay & Regional & Distant abdominal (hepatic pedicle, retroperitoneum) & Extra-abdominal \\
Liver metastases & No macroscopic nodule & Unilobar, $<5$ nodules & Bilobar and/or 5-10 nodules & $>10$ nodules \\
PC & Gilly stage 0 & Gilly stage 1/2 resectable & Gilly stage 3/4 resectable & Unresectable \\
\hline
\end{tabular}

GPS distinguishes three prognostic grades: A (0-3 points), B (4-6 points) and C (7-9 points).

impact, the dissemination type might be important because infiltrative PC is more difficult to excise surgically than nodular PC.

\section{Objectives of Surgical Treatment of NET-Derived PC}

Unlike adenocarcinomas, well-differentiated digestive NET are mostly slow growing, with an indolent course. Even at an advanced stage, and notably in the case of peritoneal involvement, an aggressive surgical approach seems to yield symptom relief and even a survival benefit $[8,19,24,25]$. Metastatic NET management is often focused solely on liver metastases, and specific PC treatment is either neglected or relegated to a secondary plan. Nevertheless, surgical removal of NET-derived PC could be justified in light of its prevalence and poor prognosis as well as the high risk of local-regional complications. PC surgery goals would include the prevention of intestinal obstruction and pain, portal hypertension and the consequences of intra-abdominal fibrosis (retractile mesenteritis and vascular involvement) [10]. Furthermore, treating PC in patients with nonresectable liver metastases would enable physicians to focus secondarily on specific treatment, including liver-directed therapies, e.g., arterial (chemo)embolization.

\section{Modalities of the Surgical Treatment of PC}

The surgical removal of PC relies on CRS, sometimes combined with HIPEC. In the most recent ENETS consensus guidelines on NET-derived PC, experts proposed that peritoneal metastases be treated aggressively in patients with favorable benefit/risk ratios, and in high-volume centers [10]. However, CRS can only be envisaged when an R0/R1 resection is expected after careful initial evaluation of the extent of PC, which relies on the thorough exploration of all abdominal quadrants. Then, CRS consists of removing all macroscopic peritoneal
Table 4. CCR status to evaluate resection completeness after cytoreductive surgery for PC (from Gilly et al. [11])

\begin{tabular}{ll}
\hline Status & Definition \\
\hline CCR-0 & No nodule visible \\
CCR-1 & Residual micronodules (size: $<2.5 \mathrm{~mm}$ ) \\
CCR-2 & Residual nodules (size: $2.5 \mathrm{~mm}$ to $<2.5 \mathrm{~cm})$ \\
CCR-3 & Residual macronodules $($ size: $>2.5 \mathrm{~cm})$ \\
\hline
\end{tabular}

metastases, potentially associated with the resection of intestinal segments, pelvic peritoneum and/or ovaries [15]. After CRS, residual PC lesions must be assessed for the completeness of cytoreduction (CCR) status, as described in table 4 [11]. The CCR status was demonstrated to be a major prognostic factor for various other malignancies and to be independent of initial PC extension [26-28]. Although this score has not been specifically applied to NET, incomplete CRS carries a poor prognosis [4].

While CRS aims to resect all visible PC and associated intra-abdominal lesions (i.e., lymph nodes and ovaries), HIPEC targets residual microscopic lesions. The efficacy of HIPEC has been demonstrated for ovarian, colorectal and gastric cancers, peritoneal mesotheliomas and pseudomyxomas $[26,28-30]$. Chemotherapy infusion into the peritoneal cavity allows it to act directly on cancer cells and achieves higher intracellular concentrations than when given intravenously [15]. Regional hyperthermia mostly affects small nonvascularized tissues (nodules) that are not protected by the cooling effect of blood circulation, thereby enhancing chemotherapeutic antitumor effects. During the procedure, a chemotherapeutic drug diluted in peritoneal dialysis solution is infused intraperitoneally, for 30-40 min, with an inflow temperature of $43^{\circ} \mathrm{C}$. The perfusion circuit is usually established with inflow and outflow percutaneous drains connected to an extracorporeal circuit in which the perfusate circu- 
lates by peristaltic pumps and is heated by a thermal exchanger. Cytotoxic agents used, alone or combined, include platin salts, irinotecan, mitomycin C, 5 -fluorouracil and doxorubicin. At the end of the procedure, the perfusate is drained and the abdomen is irrigated with normal saline $[10,15,31]$.

The efficacy of HIPEC is maximal when performed immediately after CRS, and its systemic effects are limited. Nevertheless, this procedure implies a longer operation time, is expensive and carries significant mortality and morbidity risks $(0-10 \%$ and $10-60 \%$, respectively) $[10,28]$. Extended liver resection, e.g. right hepatectomy, simultaneously with CRS and HIPEC for PC, could cause more morbidity, while minor liver resections and/or ablative treatments could be combined [ 10 , 31].

\section{Results of the Surgical Treatment of PC in NET}

The combination of CRS and HIPEC to treat NETderived PC has not been studied prospectively. In 2005, the Institut Gustave-Roussy group retrospectively reviewed 37 patients with NET-derived PC of various origins, treated with either incomplete CRS or complete CRS and liver resection followed by HIPEC [4]. For the complete CRS + HIPEC group, which had a significantly higher survival rate, the initial median PCI was 18 , with $47 \%$ of the patients experiencing grade $3 / 4$ morbidity. However, that study had inevitable selection biases; in particular, the extent of PC was not described for patients who had undergone incomplete CRS.

The same group of authors has recently updated their series [31]. Among 41 patients with PC derived from NET of various origins ( $76 \%$ small intestinal), 28 underwent complete CRS + HIPEC and 13 complete CRS alone, with similar 2-year survival rates (81 vs. $73 \%$, respectively). Their respective mortality and morbidity rates were 2 and $56 \%$. Importantly, the patients who underwent CRS + HIPEC were younger, had more frequent ileal primary NET and had lower proliferative indices; their procedures were longer, involved more resected organs and incurred greater blood losses. Although 2-year disease-free survival was significantly longer for the CRS + HIPEC group (49 vs. 17\%), the percentages of PC or liver relapses were similar to those of the CRS-treated group, indicating that adding HIPEC to CRS apparently did not delay subsequent peritoneal tumor progression.

Overall, the survival results of patients who underwent complete CRS have been better than the results of those with incomplete CRS $[4,9,19]$, even though these studies are not directly comparable (table 2). Finally, HIPEC appears to be less beneficial for patients with peritoneal metastases associated with NET than for those with colorectal cancer. Indeed, the 5-year PC relapse rate after CRS + HIPEC was about 33\% for patients with colorectal cancer $[32,33]$ but reached $55 \%$ for NET patients [31].

\section{How to Select NET Patients for PC Surgical Management}

Patients with an ileal or appendicular primary tumor location seem to be good candidates for PC surgery, since these NET are more frequently associated with PC than other primaries, and because no systemic treatments (e.g., intravenous chemotherapies and targeted therapies) have demonstrated antitumor efficacy to date. Instinctively, and as underlined by the selection bias in the paper by Elias et al. [31], patients with the lowest tumor grades or the less progressive diseases might benefit the most from PC surgery. Moreover, PC surgery should only be considered if CCR could be expected, particularly when the PCI is $<20$, for GPS-A (grade $0-3$ ) patients and some selected GPS-B (grade 4-6) patients after caseby-case assessment $[10,14]$. Evaluating the PC form could help distinguish between 'low' (i.e., nodular PC) and 'high' (fusiform/infiltrative PC) GPS-B patients. Conversely, aggressive PC surgery is contraindicated for GPS-C (grade 7-9) patients, and should not be proposed to patients with major liver metastatic involvement and/ or multiple dissemination sites (especially bone metastases), or patients in poor general condition and/or with severe comorbidities.

Importantly, aggressive surgical resection of NET-associated PC cannot be considered in an emergency context. Although patients with tumor-related symptoms (especially occlusion) tend to have more surgical interventions, operations should be undertaken with symptomatic intent only, sometimes combined with minimal cytoreduction for debulking, and almost never combined with HIPEC. Finally, the presence of hormonal symptoms per se (e.g., carcinoid syndrome) should not influence the decision to surgically resect $\mathrm{PC}$, because hormonal symptom relief is not a CRS aim. Notably, a carcinoid syndrome is usually associated with nonresectable multiple metastases, especially in the liver, which precludes PC surgery. 


\section{Conclusions}

NET-derived PC is the third most frequent NET dissemination site after the liver and distant lymph nodes and has an independent, pejorative prognostic impact on PC in patients with digestive NET. The extent of PC and its severity can be assessed by analogy to other malignancies (Gilly's score, PCI) but must be adapted to NET specificities. GPS validation is mandatory, and efforts should be made to develop non- or minimally invasive assessments of PC presence and extent.

CRS is likely to prolong survival when complete resection of the peritoneal lesions is achieved. The place of HIPEC has not yet been sufficiently explored and remains to be defined. Should a HIPEC-related survival benefit be confirmed, it will probably be short, and the procedure is associated with high morbidity. NET heterogeneity and our limited current knowledge of associated PC hamper the constitution of homogeneous patient subgroups for management. However, future studies should focus on identifying the candidates most likely to benefit from PC surgical management, according to the extent of PC and macroscopic form as well as NET characteristics including its primary localization and aggressiveness.

\section{Acknowledgement}

The authors thank Ms. Janet Jacobson for editorial assistance in the preparation of the manuscript.

\section{Disclosure Statement}

None of the authors have any personal conflicts of interest in relationship to this study, and no funding was received to support this work.

\section{References}

1 Lombard-Bohas C, Mitry E, O’Toole D, Louvet C, Pillon D, Cadiot G, Borson-Chazot F, Aparicio T, Ducreux M, Lecomte T, Etienne PL, Cacheux W, Legoux JL, Seitz JF, Ruszniewski P, Chayvialle JA, Rougier P: Thirteen-month registration of patients with gastroenteropancreatic endocrine tumors in France. Neuroendocrinology 2009;89:217-222.

2 Walter T, Scoazec J-Y, Lepage C: Epidemiology of digestive neuroendocrine tumors with focus on French data. Hépato-Gastro 2013; 20:160-166.

-3 Vasseur B, Cadiot G, Zins M, Fléjou JF, Belghiti J, Marmuse JP, Vilgrain V, Bernades P, Mignon M, Ruszniewski P: Peritoneal carcinomatosis in patients with digestive endocrine tumors. Cancer 1996;78:1686-1692.

-4 Elias D, Sideris L, Liberale G, Ducreux M, Malka D, Lasser P, Duvillard P, Baudin E: Surgical treatment of peritoneal carcinomatosis from well-differentiated digestive endocrine carcinomas. Surgery 2005;137:411-416.

$\checkmark 5$ Pape U-F, Berndt U, Müller-Nordhorn J, Böhmig M, Roll S, Koch M, Willich S, Wiedenmann B: Prognostic factors of long-term outcome in gastroenteropancreatic neuroendocrine tumours. Endocr Relat Cancer 2008; 15:1083-1097.

6 Strosberg J, Gardner N, Kvols L: Survival and prognostic factor analysis of 146 metastatic neuroendocrine tumors of the mid-gut. Neuroendocrinology 2009;89:471-476.

7 Bhosale P, Shah A, Wei W, Varadhachary G, Johnson V, Shah V, Kundra V: Carcinoid tumours: predicting the location of the primary neoplasm based on the sites of metastases. Eur Radiol 2013;23:400-407.
8 Chambers AJ, Pasieka JL, Dixon E, Rorstad O: The palliative benefit of aggressive surgical intervention for both hepatic and mesenteric metastases from neuroendocrine tumors. Surgery 2008;144:645-653.

-9 Norlén O, Stålberg P, Öberg K, Eriksson J, Hedberg J, Hessman O, Janson ET, Hellman P, Åkerström G: Long-term results of surgery for small intestinal neuroendocrine tumors at a tertiary referral center. World J Surg 2012; 36:1419-1431.

10 Kianmanesh R, Ruszniewski P, Rindi G, Kwekkeboom D, Pape U-F, Kulke M, Sevilla Garcia I, Scoazec JY, Nilsson O, Fazio N, Lesurtel M, Chen YJ, Eriksson B, Cioppi F, O'Toole D: ENETS consensus guidelines for the management of peritoneal carcinomatosis from neuroendocrine tumors. Neuroendocrinology 2010;91:333-340.

11 Gilly FN, Cotte E, Brigand C, Monneuse O, Beaujard AC, Freyer G, Glehen O: Quantitative prognostic indices in peritoneal carcinomatosis. Eur J Surg Oncol 2006;32:597-601.

-12 Jacquet P, Sugarbaker PH: Clinical research methodologies in diagnosis and staging of patients with peritoneal carcinomatosis. Cancer Treat Res 1996;82:359-374.

-13 Swellengrebel HAM, Zoetmulder FAN, Smeenk RM, Antonini N, Verwaal VJ: Quantitative intra-operative assessment of peritoneal carcinomatosis: a comparison of three prognostic tools. Eur J Surg Oncol 2009;35: 1078-1084

14 Au JT, Levine J, Aytaman A, Weber T, Serafini F: Management of peritoneal metastasis from neuroendocrine tumors. J Surg Oncol 2013;108:385-386.
5 Brücher B, Piso P, Verwaal V, Esquivel J, Derraco $\mathrm{M}$, Yonemura $\mathrm{Y}$, Gonzalez-Moreno $\mathrm{S}$, Pelz J, Königsrainer A, Ströhlein M, Levine E, Morris D, Bartlett D, Glehen O, Garofalo A, Nissan A: Peritoneal carcinomatosis: cytoreductive surgery and HIPEC: overview and basics. Cancer Invest 2012;30:209-24.

16 Rivard J, Temple W, McConnell Y, Sultan H, Mack L: Preoperative computed tomography does not predict resectability in peritoneal carcinomatosis. Am J Surg 2014;207:760-765.

17 Sommariva A, Zagonel V, Rossi CR: The role of laparoscopy in peritoneal surface malignancies selected for hyperthermic intraperitoneal chemotherapy (HIPEC). Ann Surg Oncol 2012;19:3737-3744.

18 Iversen LH, Rasmussen PC, Laurberg S: Value of laparoscopy before cytoreductive surgery and hyperthermic intraperitoneal chemotherapy for peritoneal carcinomatosis. $\mathrm{Br} \mathrm{J}$ Surg 2013;100:285-292.

19 Boudreaux JP, Putty B, Frey DJ, Woltering E, Anthony L, Daly I, Ramcharan T, Lopera J, Castaneda W: Surgical treatment of advanced-stage carcinoid tumors: lessons learned. Ann Surg 2005;241:839-845.

20 Makridis C, Rastad J, Oberg K, Akerström G: Progression of metastases and symptom improvement from laparotomy in midgut carcinoid tumors. World J Surg 1996;20:900-906.

21 Ohrvall U, Eriksson B, Juhlin C, Karacagil S, Rastad J, Hellman P, Åkerström G: Method for dissection of mesenteric metastases in mid-gut carcinoid tumors. World J Surg 2000; 24:1402-1408. 
22 Hellman P, Lundström T, Ohrvall U, Eriksson B, Skogseid B, Oberg K, Janson ET, Åkerström G: Effect of surgery on the outcome of midgut carcinoid disease with lymph node and liver metastases. World J Surg 2002;26: 991-997.

23 Akerström G, Hellman P, Hessman O: Midgut carcinoid tumours: surgical treatment and prognosis. Best Pract Res Clin Gastroenterol 2005;19:717-728.

-24 de Mestier L, Neuzillet C, Hentic O, Kianmanesh R, Hammel P, Ruszniewski P: Prolonged survival in a patient with neuroendocrine tumor of the cecum and diffuse peritoneal carcinomatosis. Case Rep Gastroenterol 2012;6:205-210.

25 Elias D, Lasser P, Ducreux M, Duvillard P, Ouellet J-F, Dromain C, Schlumberger M, Pocard M, Boige V, Miquel C, Baudin E: Liver resection (and associated extrahepatic resections) for metastatic well-differentiated endocrine tumors: a 15-year single center prospective study. Surgery 2003;133:375-382.
26 Glehen O, Gilly FN, Boutitie F, Bereder JM, Quenet F, Sideris L, Mansvelt B, Lorimier G, Msika S, Elias D: Toward curative treatment of peritoneal carcinomatosis from nonovarian origin by cytoreductive surgery combined with perioperative intraperitoneal chemotherapy: a multi-institutional study of 1,290 patients. Cancer 2010;116:5608-5618.

27 Verwaal VJ, van Ruth S, de Bree E, van Sloothen GW, van Tinteren H, Boot H, Zoetmulder F: Randomized trial of cytoreduction and hyperthermic intraperitoneal chemotherapy versus systemic chemotherapy and palliative surgery in patients with peritoneal carcinomatosis of colorectal cancer. J Clin Oncol 2003;21:3737-3743.

28 Elias D, Goéré D, Dumont F, Honoré C, Dartigues P, Stoclin A, Malka D, Boige V, Ducreux M: Role of hyperthermic intraoperative peritoneal chemotherapy in the management of peritoneal metastases. Eur J Cancer 2014; 50:332-340.

29 Glehen O, Mohamed F, Gilly FN: Peritoneal carcinomatosis from digestive tract cancer: new management by cytoreductive surgery and intraperitoneal chemohyperthermia. Lancet Oncol 2004;5:219-228.
30 Glockzin G, Schlitt HJ, Piso P: Peritoneal carcinomatosis: patients selection, perioperative complications and quality of life related to cytoreductive surgery and hyperthermic intraperitoneal chemotherapy. World J Surg Oncol 2009;7:5.

31 Elias D, David A, Sourrouille I, Honoré C, Goéré D, Dumont F, Stoclin A, Baudin E: Neuroendocrine carcinomas: optimal surgery of peritoneal metastases (and associated intra-abdominal metastases). Surgery 2014;155: 5-12.

32 Elias D, Benizri E, Di Pietrantonio D, Menegon P, Malka D, Raynard B: Comparison of two kinds of intraperitoneal chemotherapy following complete cytoreductive surgery of colorectal peritoneal carcinomatosis. Ann Surg Oncol 2007;14:509-514.

33 Quenet F, Goéré D, Mehta SS, Roca L, Dumont F, Hessissen M, Saint-Aubert B, Elias D: Results of two bi-institutional prospective studies using intraperitoneal oxaliplatin with or without irinotecan during HIPEC after cytoreductive surgery for colorectal carcinomatosis. Ann Surg 2011;254:294-301. 